ORIGINAL ARTICLE

\title{
Expression of mismatch repair proteins, $\beta$ catenin, and $E$ cadherin in intestinal-type sinonasal adenocarcinoma
}

\author{
B Perez-Ordonez, N N Huynh, K W Berean, R C K Jordan
}

J Clin Pathol 2004;57:1080-1083. doi: 10.1136/jcp.2004.018051

See end of article for authors' affiliations

.....................

Correspondence to: Dr B Perez-Ordoñez Department of Pathology University Health Network, Princess Margaret Hospital, 610 University Avenue, Room 4-304, Toronto, Ontario M4N 3M5, Canada; bayardo. perez-ordonez@uhn.on.ca

Accepted for publication 21 April 2004
Background: Despite their histological resemblance to colorectal adenocarcinomas, there is little information about the molecular events involved in the pathogenesis of intestinal-type sinonasal adenocarcinomas (ITACs).

Aims: To evaluate the possible role of DNA mismatch repair (MMR) gene defects or disruptions of the $E$ cadherin $-\beta$ catenin complex in ITAC by investigating the immunohistochemical expression of the MMR gene products, $\beta$ catenin, and $E$ cadherin in a group of sporadic ITACs.

Methods: Ten sporadic cases of ITAC were stained with antibodies against MLH1, MSH2, MSH3, MSH6, $\beta$ catenin, and $E$ cadherin.

Results: Nine cases showed strong nuclear expression of $\mathrm{MLH1}$, whereas one case showed moderate staining. All 10 cases were strongly positive for MSH2 and MSH3. MSH6 was strong in nine cases, and moderate in one. Membranous $\beta$ catenin expression was strong in all 10 cases, and no case showed cytoplasmic or nuclear staining. E cadherin was strong in seven cases, and moderate in three cases.

Conclusions: The preserved nuclear expression of MLH1, MSH2, MSH3, and MSH6 suggests that mutations or promoter methylation of MMR genes do not play a role in the pathogenesis of ITAC. The strong membranous staining for $E$ cadherin and $\beta$ catenin and lack of abnormal cytoplasmic or nuclear expression is in keeping with the preservation of $E$ cadherin $\beta$ catenin complexes and $\beta$ catenin pathways. ntestinal-type adenocarcinomas (ITACs) of the nasal cavity and paranasal sinuses are rare tumours that occur sporadically or are associated with occupational exposure to hardwood and leather dusts, nickel, and possibly smoking. ${ }^{2}$ ITACs are aggressive tumours characterised by frequent local recurrences, low incidence of distant metastases, and an overall mortality of approximately $53 \% .^{2}$ The morphological appearance of these tumours is variable and they may resemble normal small intestinal mucosa, villous adenomas, conventional colorectal adenocarcinomas, mucinous adenocarcinomas, and signet ring cell carcinomas. ${ }^{1-3}$ The similarities between ITAC and intestinal adenocarcinoma extend to their ultrastructural and immunohistochemical characteristics. ${ }^{3-6}$

"In recent years, immunohistochemistry for MLHI and MSH2 nuclear protein expression has been shown to be useful as a primary screening test for mismatch repair gene defects"

Unlike ITAC, colorectal adenocarcinoma is one of the most common and widely studied human malignancies. ${ }^{78}$ Numerous studies have shown that mutations of the K-ras, p53, and APC (adenomatous polyposis coli) genes are common in colorectal adenocarcinomas. ${ }^{78}$ In comparison, there is very little information about the molecular events involved in the pathogenesis of ITAC. Working on the hypothesis that morphological similarities with colorectal adenocarcinomas might reflect equivalent genetic alterations, several authors have investigated the presence of p53 mutations and the activation of ras oncogenes in ITAC. ${ }^{9-12}$ However, $\mathrm{Wu}$ and colleagues ${ }^{11}$ found p53 mutations in only two of 11 ITACs, with no cases showing K-ras mutations. In a report composed mainly of occupational tumours from Scandinavia, Saber et al described K-ras mutations in four of 28 cases. $^{9}$ Perez and co-workers ${ }^{10}$ studied the status of three ras oncogenes in 31 cases of ethmoid sinus ITAC. H-ras mutations were found in only five of the 31 patients. One additional patient had mutations of K-ras and there were no $\mathrm{N}$-ras gene activations. In contrast to the report by Wu et al, ${ }_{1}^{11}$ Perrone and colleagues ${ }^{12}$ demonstrated p53 mutations in $40 \%$ of mostly occupational ITACs. These authors also found a high prevalence of loss of heterozygosity at the $17 \mathrm{p} 13$ locus $(64 \%)$, in addition to pl4 ${ }^{\mathrm{ARF}}(67 \%)$ and $\mathrm{pl6}^{\mathrm{INK} 4 \mathrm{a}}(69 \%)$ promoter methylation. ${ }^{12}$

Approximately $90 \%$ of carcinomas arising in patients with hereditary non-polyposis colorectal adenocarcinoma and 10$15 \%$ of sporadic colorectal adenocarcinomas are associated with the phenomenon of microsatellite instability (MSI) and mutations or promoter methylation in the MLH1 and MSH2 mismatch repair (MMR) genes. ${ }^{8}{ }^{13}$ Other MMR genes include MSH3, MSH6, PMS1, and PMS2. These genes play a smaller role in the pathogenesis of hereditary non-polyposis colorectal adenocarcinoma and sporadic colorectal adenocarcinomas. MSI and defects in the MMR genes are generally investigated by DNA microsatellite analysis or gene sequencing. However, these DNA techniques are expensive and labour intensive. In recent years, immunohistochemistry (IHC) for MLH1 and MSH2 nuclear protein expression has been shown to be useful as a primary screening test for MMR gene defects. ${ }^{14}{ }^{15}$ In a large study by Lindor et al, ${ }^{14}$ IHC showed a $100 \%$ specificity and $92 \%$ sensitivity in classifying colorectal adenocarcinomas for MMR competency. Based on the histological similarities between colorectal carcinoma and ITACs, and the reported low prevalence of K-ras mutations and somewhat conflicting data regarding p53 status in ITACs, we explored the possibility that MSI and the DNA mismatch repair pathway are involved in the pathogenesis of ITAC. For

Abbreviations: APC, adenomatous polyposis coli; IHC, immunohistochemistry; ITAC, intestinal-type sinonasal adenocarcinoma; $M M R$, mismatch repair; MSI, microsatellite instability 
Table 1 Details of the antibodies used in our study

\begin{tabular}{lllll}
\hline Antigen & Source & Clone & Dilution & Buffer \\
\hline Human MLH1 & BD Biosciences, Mississauga, & G168-728 & $1 / 50$ & Citrate pH 6.0 \\
& Canada & G219-1129 & $1 / 200$ & Citrate pH 6.0 \\
Human MSH2 & BD Biosciences & 52 & $1 / 100$ & Citrate $\mathrm{pH} 6.0$ \\
Human MSH3 & BD Biosciences & 44 & $1 / 25$ & Citrate $\mathrm{pH} 6.0$ \\
Human MSH6 & BD Biosciences & 14 & $1 / 500$ & Citrate pH 6.0 \\
$\beta$ Catenin & BD Biosciences & HECD-1 & $1 / 200$ & Citrate pH 6.0 \\
E cadherin & Zymed, South San Francisco, & & \\
& California, USA & & & \\
& & &
\end{tabular}

this purpose, we used a panel of monoclonal antibodies against the MLH1, MSH2, MSH3, and MSH6 gene products.

In addition, because mutations of the APC gene, found in approximately $70 \%$ of sporadic colorectal adenocarcinomas, ${ }^{8}$ and activating mutations in the $\beta$ catenin gene lead to the accumulation of $\beta$ catenin in the cytoplasm and nucleus of colorectal tumour cells, ${ }^{16}$ we also explored the possibility of dysfunction of $E$ cadherin- $\beta$ catenin complexes in ITAC by IHC using antibodies for $\beta$ catenin and E cadherin.

\section{MATERIAL AND METHODS}

All 10 cases were retrieved from surgical pathology files on the basis of an original pathological diagnosis of sinonasal ITAC. Original haematoxylin and eosin stained slides were reviewed by two of the authors (BP-O, $\mathrm{NNH}$ ) and the original diagnoses were confirmed in all cases. A representative formalin fixed, paraffin wax embedded block from each case was selected for immunohistochemical studies using the antibodies listed in table 1. All immunostaining was performed manually with microwave antigen retrieval and overnight incubation with the primary antibodies. The staining was completed using the ultrastreptavidin-horseradish peroxidase detection system (ID Laboratories Biotechnology, London, Ontario, Canada) and colour development was performed using the NovaRed substrate kit (Vector Laboratories, Burlingame, California, USA). Sections of normal colon and sporadic colonic adenocarcinomas were used as positive controls, respectively. The expression of MLH1, MSH2, MSH3, and MSH6 was considered positive if there was definitive nuclear staining in any of the tumour cells. Beta catenin and E cadherin staining were considered positive if clear membrane immunoreactivity was present. Special attention was paid to the presence of aberrant cytoplasmic and nuclear expression of $\beta$ catenin. The

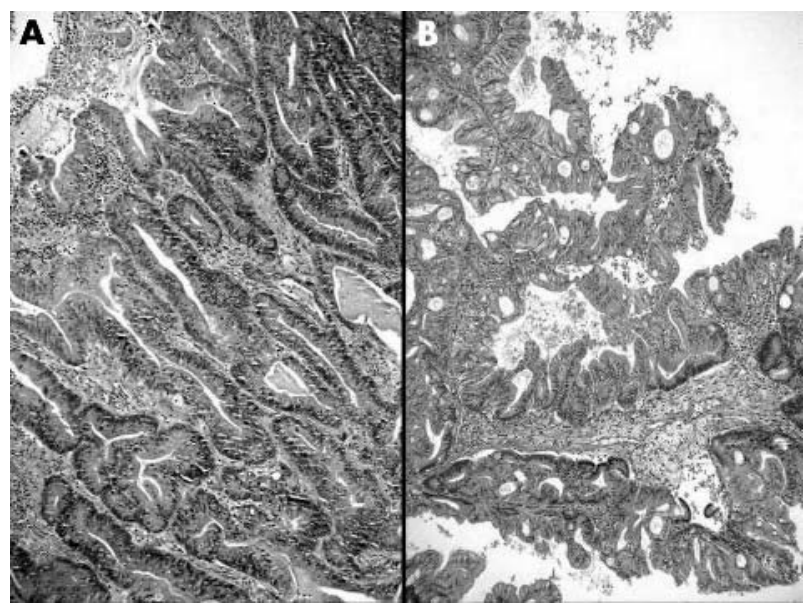

Figure 1 (A) Colonic intestinal-type sinonasal adenocarcinoma (ITAC) and (B) papillary-type ITAC. immunohistochemical stains were evaluated semiquantitatively and scored as: negative ( $0 \%$ positive cells), mild $(>1-$ $25 \%$ positive cells), moderate ( $>25-50 \%$ positive cells), and strong ( $>50 \%$ positive cells).

\section{RESULTS}

Nine patients were male and one was female. None of the patients had a known history of occupational exposure to hardwood dust. The age at presentation ranged from 21 to 87 years (mean, 63). Five tumours arose in the ethmoid sinus, three in the maxillary sinus, one in the nasal cavity, and one in the sphenoid sinus.

The 10 cases of ITAC comprised five colonic type (fig lA), four papillary (fig 1B), and one mixed with papillary architecture and abundant extracellular mucin production. Table 2 summarises the immunohistochemical results. Nine cases showed strong nuclear expression of MLHl (fig 2A), whereas one case showed moderate staining. All 10 cases were strongly positive for MSH2 and MSH3 (figs 2B, C). MSH6 was strong in nine cases and moderate in one (fig 2D). All cases showed membrane staining for E cadherin and $\beta$ catenin. E cadherin staining was strong in seven cases (fig 3A) and moderate in three cases. Beta catenin expression was strong in all 10 cases (fig 3B). No case showed cytoplasmic or nuclear staining for $\beta$ catenin or E cadherin.

\section{DISCUSSION}

Despite their histological similarity to colorectal carcinomas, there is little information about the molecular events involved in the pathogenesis of ITACs. Several crucial pathways of tumorigenesis have been identified in colorectal adenocarcinomas. ${ }^{78}$ These pathways involve the mutation and inactivation of multiple oncogenes, tumour suppressor genes, and DNA mismatch repair genes including K-ras, APC, p53, MLHl, and MSH2. ${ }^{73}$ Working on the hypothesis that morphological similarities to colorectal adenocarcinomas might reflect equivalent genetic alterations, several authors have investigated the presence of p53 mutations and activating mutations of ras oncogenes in ITAC. ${ }^{9-12}$ However, the results of these studies suggest that mutations of K-ras and other ras genes are relatively uncommon in ITAC. ${ }^{9-12}$ Similarly, p53 mutations in ITACs have not been widely demonstrated. ${ }^{11}{ }^{12} \mathrm{Wu}$ et al found p53 mutations in only two of 11 cases investigated. ${ }^{11}$ More recently Perrone et al found p53 mutations in eight of 20 cases of mostly occupational ITACs. ${ }^{12}$ Given these findings, we have explored the possibility that different pathways involved in colorectal carcinogenesis-namely, the DNA mismatch repair and the APC $-\beta$ catenin pathways-are involved in the molecular pathogenesis of ITACs. For this purpose, we used a panel of antibodies against MLH1, MSH2, MSH3, MSH6, $\beta$ catenin, and E cadherin.

There are no previous reports on the expression of MMR proteins in ITACs. All of our cases showed preserved nuclear expression of MLH1, MSH2, MSH3, and MSH6, with most 

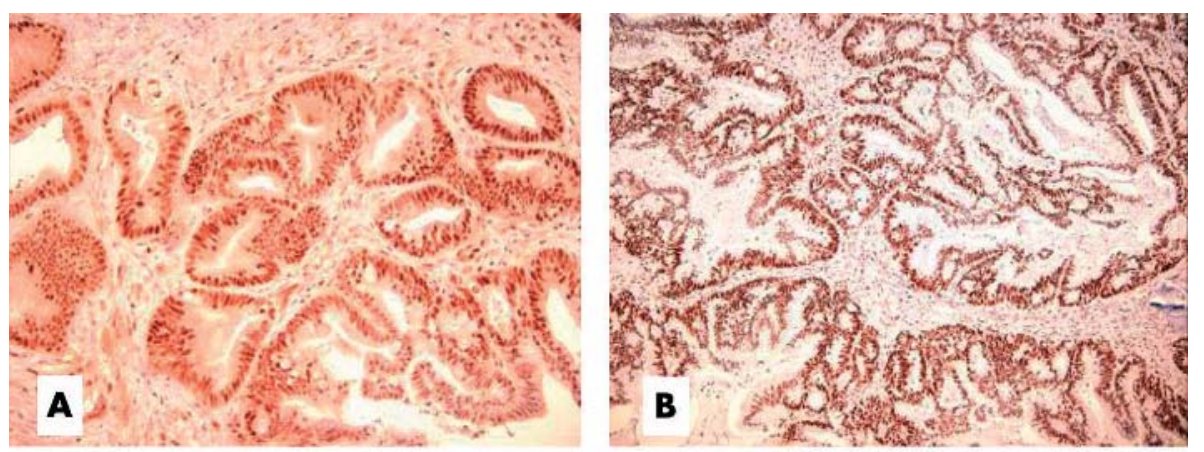

Figure 2 Intestinal-type sinonasal adenocarcinomas with strong nuclear staining for (A) MLH1, (B) MSH2, (C) $\mathrm{MSH} 3$, and (D) MSH6.
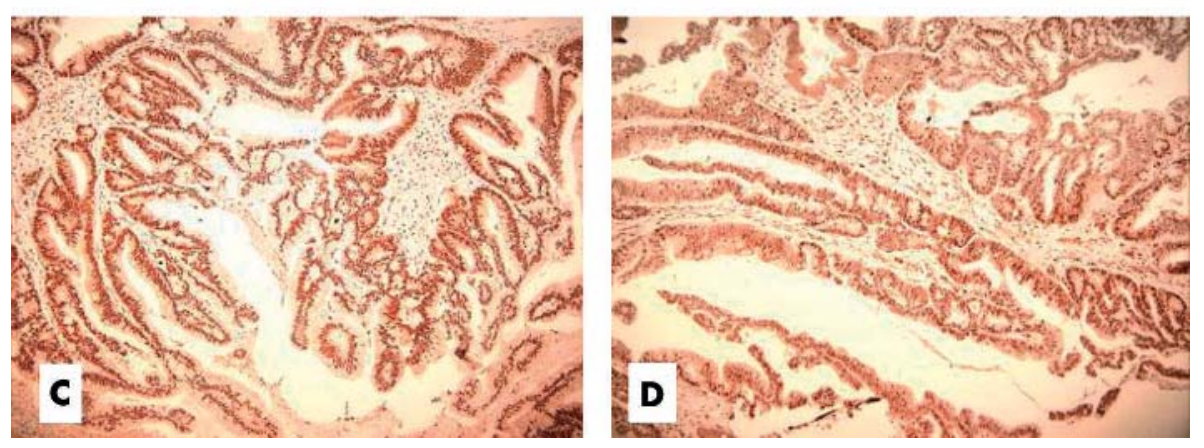

cases showing strong nuclear staining. These results suggest that mutations or promoter methylation of these MMR genes do not play an important role in the pathogenesis of ITAC. Although we did not perform microsatellite analysis or gene sequencing, it has been shown that IHC is highly sensitive and specific for the preliminary screening of MMR gene defects in colorectal adenocarcinomas. ${ }^{14} 1517$ Thibodeau et al reported that all tumours classified as microsatellite stable and low level microsatellite instability had preserved nuclear expression of MLHI or MSH2 proteins. ${ }^{17}$ Marcus et al reported similar results in a smaller study. ${ }^{15}$ In a study involving 1144 patients, ${ }^{14}$ IHC for MLHI and MSH2 showed a $100 \%$ specificity and $92 \%$ sensitivity in classifying colorectal adenocarcinomas for MMR competency. That study showed that absence of MLH1 and MSH2 expression was 100\% accurate for predicting a tumour with high MSI, whereas only 3.3\% (27 of 818 ) of tumours with normal expression for both markers had a high level of MSI by molecular testing with 10 markers. ${ }^{14}$ Despite the high sensitivity of IHC in the
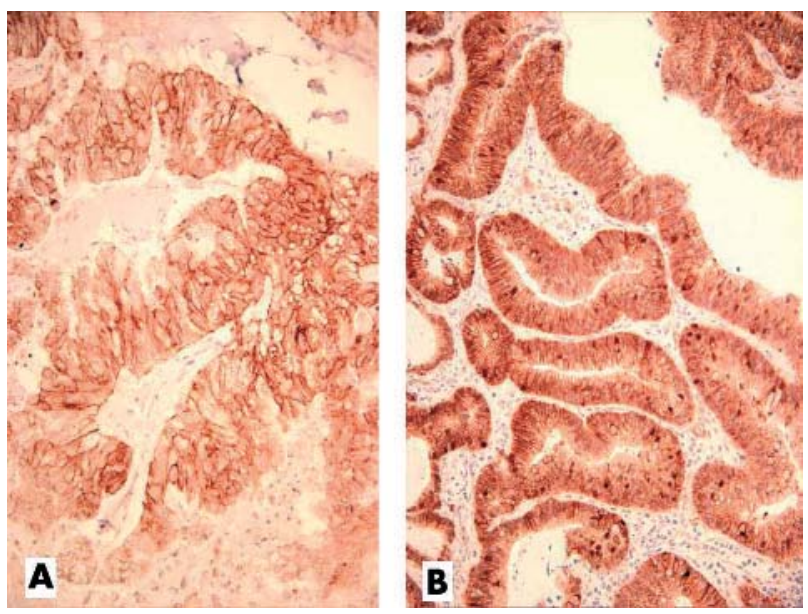

Figure 3 Intestinal-type sinonasal adenocarcinoma with typical diffuse membranous staining for (A) $E$ cadherin and (B) $\beta$ catenin. preliminary screening of MMR gene defects in colorectal adenocarcinomas, there are occasionally discrepant results between preserved MMR protein expression and MMR mutational gene status in a small number of tumours. ${ }^{19} 20$ In general, these discrepancies are the result of rare in frame deletions of MSH2 or in frame deletions or splice mutations in exon 16 and exon 19 of the MLHl gene, resulting in the production of large fragments or full length proteins. ${ }^{19} 20$ There is less experience in the use of IHC to assess MSH3 and MSH6 expression ${ }^{19} 2122$; however, preserved nuclear expression of these proteins generally indicates lack of pathogenetic mutations in these genes.

"Our results suggest that unlike conventional intestinal adenocarcinomas, there are no alterations in the E cadherin-catenin complexes or the $\beta$ catenin pathways in intestinal-type sinonasal adenocarcinomas"

E cadherin is a cell adhesion molecule with an important role in intercellular adhesion. The cytoplasmic domain of E cadherin binds to $\beta$ catenin and $\gamma$ catenin. ${ }^{23}$ These E cadherin-catenin complexes interact with the actin cytoskeleton through associations with $\alpha$ catenin, $\alpha$ actinin, and actin filaments. Alterations in E cadherin expression and

\begin{tabular}{|c|c|c|c|c|c|c|}
\hline Case & MLH-1 & MSH-2 & MSH-3 & MSH-6 & E cadherin & $\beta$ Catenin \\
\hline 1 & $3+$ & $3+$ & $3+$ & $3+$ & $3+$ & $3+$ \\
\hline 2 & $3+$ & $3+$ & $3+$ & $3+$ & $2+$ & $3+$ \\
\hline 3 & $3+$ & $3+$ & $3+$ & $2+$ & $3+$ & $3+$ \\
\hline 4 & $2+$ & $3+$ & $3+$ & $3+$ & $3+$ & $3+$ \\
\hline 5 & $3+$ & $3+$ & $3+$ & $3+$ & $3+$ & $3+$ \\
\hline 6 & $3+$ & $3+$ & $3+$ & $3+$ & $2+$ & $3+$ \\
\hline 7 & $3+$ & $3+$ & $3+$ & $3+$ & $3+$ & $3+$ \\
\hline 8 & $3+$ & $3+$ & $3+$ & $3+$ & $2+$ & $3+$ \\
\hline 9 & $3+$ & $3+$ & $3+$ & $3+$ & $3+$ & $3+$ \\
\hline 10 & $3+$ & $3+$ & $3+$ & $3+$ & $3+$ & $3+$ \\
\hline
\end{tabular}

$3+$, strong staining; $2+$, moderate staining; $1+$, mild staining; - , negative. 


\section{Take home messages}

- We found that the nuclear expression of the MLHI $\mathrm{MSH} 2, \mathrm{MSH} 3$, and MSH6 genes was preserved in intestinal-type sinonasal adenocarcinomas (ITACs)

- This suggests that, unlike colorectal adenocarcinomas, which they resemble histologically, mutations or promoter methylation of DNA mismatch repair genes and microsatellite instability do not play a role in the pathogenesis of these tumours

- The strong membranous staining for $E$ cadherin and $\beta$ catenin and the lack of abnormal cytoplasmic or nuclear expression indicate that, unlike conventional intestinal adenocarcinomas, there are no alterations in the $E$ cadherin-catenin complexes or the $\beta$ catenin pathways in ITACs

function are common in intestinal adenocarcinomas, and are usually seen as decreased membranous expression with abnormal cytoplasmic accumulation. ${ }^{24} 25$ Beta catenin also plays an important role in colorectal carcinogenesis via its interaction with numerous molecules, such as E-cadherin, the APC gene product, GSK-3 $\beta$, AXIN, and the ATC/LEF family of transcription factors. ${ }^{16}$ Mutations of the APC, CTNNB1, and AXIN2 genes seen in intestinal adenocarcinomas lead to abnormal cytoplasmic and nuclear expression of $\beta$ catenin as a result of inadequate proteolysis. ${ }^{16}$ There are no previous reports on the expression of E cadherin and $\beta$ catenin in ITACs. All cases that we investigated showed normal membranous expression with no abnormal cytoplasmic or nuclear accumulation of these proteins. Our results suggest that unlike conventional intestinal adenocarcinomas, there are no alterations in the E cadherin-catenin complexes or the $\beta$ catenin pathways in ITACs.

In conclusion, the preserved nuclear expression of MLH1, MSH2, MSH3, and MSH6 in ITACs suggests that mutations or promoter methylation of MMR genes and MSI do not play a role in the pathogenesis of these tumours. The strong membranous staining for $\mathrm{E}$ cadherin and $\beta$ catenin and lack of abnormal cytoplasmic or nuclear expression is in keeping with preserved $\mathrm{E}$ cadherin- $\beta$ catenin complexes and $\beta$ catenin pathways.

\section{Authors' affiliations}

B Perez-Ordonez, N N Huynh, Department of Pathology, University Health Network, Princess Margaret Hospital, 610 University Avenue, Room 4-304, Toronto, Ontario M4N 3M5, Canada

K W Berean, Department of Pathology, Vancouver Hospital, Vancouver, British Columbia, Canada

R C K Jordan, University of California San Francisco, San Francisco, California, USA

\section{REFERENCES}

1 Kleinsasser $\mathbf{O}$, Schroeder H-G. Adenocarcinomas of the inner nose after exposure to wood dust. Morphological findings and relationships between histopathology and clinical behavior in 79 cases. Arch Otolaryngol 1988;245:1-15.

2 Barnes L. Intestinal-type adenocarcinoma of the nasal cavity and paranasal sinuses. Am J Surg Pathol 1986;10:192-202.

3 Mills SE, Fechner RE, Cantrell RW. Aggressive sinonasal lesion resembling normal intestinal mucosa. Am J Surg Pathol 1982;6:803-9.

4 Batsakis JG, Mackay B, Ordonez NG. Enteric-type adenocarcinoma of the nasal cavity. An electron microscopic and immunocytochemical study. Cancer 1984;54:855-60.

5 McKinney CD, Mills SE, Franquemont DW. Sinonasal intestinal-type adenocarcinoma: immunohistochemical profile and comparison with colonic adenocarcinoma. Mod Pathol 1995;8:421-5.

6 Franchi A, Massi D, Baroni G, et al. CDX-2 homeobox gene expression. Am J Surg Pathol 2003;27:1390-1.

7 Fearon ER, Vogelstein B. A genetic model for colorectal tumorigenesis. Cell 1990;61:759-67.

8 Chung DC. The genetic basis of colorectal cancer: insights into critical pathways of tumorigenesis. Gastroenterology 2000;1 19:854-65.

9 Saber AT, Nielsen LR, Dictor M, et al. K-ras mutations in sinonasal adenocarcinomas in patients occupationally exposed to wood or leather dust. Cancer Lett 1998;126:59-65.

10 Perez P, Dominguez O, Gonzalez S, et al. RAS gene mutations in ethmoid sinus adenocarcinoma: prognostic implications. Cancer 1999;86:255-64.

11 Wu T-T, Barnes L, Bakker A, et al. K-ras-2 and p53 genotyping of intestinaltype adenocarcinoma of the nasal cavity and paranasal sinuses. Mod Pathol 1996;9:199-204.

12 Perrone F, Oggionni M, Birindelli S, et al. TP53, pl4ARF, p16INK4a and Hras gene molecular analysis in intestinal-type adenocarcinoma of the nasal cavity and paranasal sinuses. Int J Cancer 2003;105:196-203.

13 Calvert PM, Frucht H. The genetics of colorectal cancer. Ann Intern Med 2002;137:603-12.

14 Lindor NM, Burgart $\amalg$, Leontovich $O$, et al. Immunohistochemistry versus microsatellite instability testing in phenotyping colorectal tumors. J Clin Oncol 2002;20:1043-8.

15 Marcus VA, Madlensky L, Gryfe R, et al. Immunohistochemistry for hMLH1 and hMSH2: a practical test for DNA mismatch repair-deficient tumors. Am J Surg Pathol 1999;23:1248-55.

16 Wong NA, Pignatelli M. Beta-catenin-a linchpin in colorectal carcinogenesis? Am J Pathol 2002;160:389-401.

17 Thibodeau SN, French AJ, Cunningham JM, et al. Microsatellite instability in colorectal cancer: different mutator phenotypes and the principal involvement of hMLH1. Cancer Res 1998;58:1713-18.

18 De Jong AE, Van Puijenbroek M, Hendriks Y, et al. Microsatellite instability, immunohistochemistry, and additional PMS2 staining in suspected hereditary nonpolyposis colorectal cancer. Clin Cancer Res 2004;10:972-80.

19 Hendriks Y, Franken P, Dierssen JW, et al. Conventional and tissue microarray immunohistochemical expression analysis of mismatch repair in hereditary colorectal tumors. Am J Pathol 2003;162:469-77.

20 Wahlberg SS, Schmeits J, Thomas G, et al. Evaluation of microsatellite instability and immunohistochemistry for the prediction of germ-line MSH2 and MLH1 mutations in hereditary nonpolyposis colon cancer families. Cancer Res 2002;62:3485-92.

21 Rigau V, Sebbagh N, Olschwang S, et al. Microsatellite instability in colorectal carcinoma. The comparison of immunohistochemistry and molecular biology suggests a role for hMSH6 immunostaining. Arch Pathol Lab Med 2003; 127:694-700.

22 Plaschke J, Kruger S, Jeske B, et al. Loss of MSH3 protein expression is frequent in $\mathrm{MLH1}$-deficient colorectal cancer and is associated with disease progression. Cancer Res 2004;64:864-70.

23 Smith MEF, Pignatelli $M$. The molecular histology of neoplasia: the role of the cadherin/catenin complex. Histopathology 1997;31:107-11.

24 El Bahrawy MA, Poulsom R, Jeffery $R$, et al. The expression of E-cadherin and catenins in sporadic colorectal carcinoma. Hum Pathol 2001;32:1216-24.

25 Wheeler JM, Warren BF, Mortensen NJ, et al. An insight into the genetic pathway of adenocarcinoma of the small intestine. Gut 2002;50:218-23. 